\title{
Thermodynamics of Abelian Gauge Fields in Real Hyperbolic Spaces
}

\author{
A. A. Bytsenko \\ Departamento de Física, Universidade Estadual de Londrina, \\ Caixa Postal 6001, Londrina-Parana, Brazil E-mail address: abyts@uel.br \\ V. S. Mendes \\ Departamento de Física, Universidade Estadual de Londrina, Caixa Postal 6001, \\ Londrina-Parana, Brazil E-mail address: vsmendes@yahoo.com.br
}

A. C. Tort

Departamento de Física Teórica - Instituto de Fúsica Universidade Federal do Rio de Janeiro, Caixa postal 68.528; CEP 21941-972, Rio de Janeiro, Brazil E-mail address: tort@if.ufrj.br

(Dated: November, 2003)

\begin{abstract}
We work with $N$-dimensional compact real hyperbolic space $X_{\Gamma}$ with universal covering $M$ and fundamental group $\Gamma$. Therefore, $M$ is the symmetric space $G / K$, where $G=S O_{1}(N, 1)$ and $K=S O(N)$ is a maximal compact subgroup of $G$. We regard $\Gamma$ as a discrete subgroup of $G$ acting isometrically on $M$, and we take $X_{\Gamma}$ to be the quotient space by that action: $X_{\Gamma}=\Gamma \backslash M=\Gamma \backslash G / K$. The natural Riemannian structure on $M$ (therefore on $X$ ) induced by the Killing form of $G$ gives rise to a connection $p$-form Laplacian $\mathfrak{L}_{p}$ on the quotient vector bundle (associated with an irreducible representation of $\mathrm{K}$ ). We study gauge theories based on abelian $p$-forms on the real compact hyperbolic manifold $X_{\Gamma}$. The spectral zeta function related to the operator $\mathfrak{L}_{p}$, considering only the co-exact part of the $p$-forms and corresponding to the physical degrees of freedom, can be represented by the inverse Mellin transform of the heat kernel. The explicit thermodynamic fuctions related to skew-symmetric tensor fields are obtained by using the zeta-function regularization and the trace tensor kernel formula (which includes the identity and hyperbolic orbital integrals). Thermodynamic quantities in the high and low temperature expansions are calculated and new entropy/energy ratios established.
\end{abstract}

PACS numbers: 04.70.Dy, 11.25.Mj

\section{INTRODUCTION}

It is believed that the real universe is a form of Friedman-Robertson-Walker models. However, the timedependent metrics of these models brings about at least two inconvenient problems: (i) the definition of vacuum state in a time-dependent background; the background starts producing particles in a continuous way, and therefore, vacuum states in the Minkowskian sense do not exist; (ii) the all-important thermodynamic investigation of the early universe becomes senseless due to the lack of a proper definition of equilibrium state [1]. Einstein static universes can meet the inconveniences stated before. For these type of manifold, a vacuum state can be unambiguously defined both locally and globally. It can be shown that a closed universe has the same vacuum energy and pressure as a static Einstein universe [2]. The radius of this universe is the instantaneous radius of the Friedman-Robertson-Walker universe. Finitetemperature field theory in curved backgrounds makes sense again. The thermodynamics of quantum fields in an Einstein universe for some radius is equivalent to that of an instantaneously static closed universe. The thermodynamics of positive curvature Einstein spaces was discussed by several authors before. In particular the socalled entropy bounds or entropy to thermal energy ratios were calculated and compared with known bounds such as the Bekenstein bound or the Cardy-Verlinde bound. For example, for a massless scalar field in $\mathbb{S}^{3}$ space this was done in [3] and for a massive scalar field in [4]. Here we wish to extend the evaluation of those type of bounds to the case of skew-symmetric tensor fields in real compact hyperbolic spaces, a more delicate and involved task when compared with previous calculations in $\mathbb{S}^{3}$ space, as we shall see. This paper is divided as follows: In Section II we set the relevant mathematical tools of the quantum dynamics of exterior forms of real hyperbolic spaces; in Section III we apply Fried trace formula to the tensor kernel and derive the identity and the hyperbolic contributions; in Section IV the spectral functions associated with exterior forms are obtained; in Sections V and VI we establish the pertinent high and low temperature expansions and calculate the entropy bounds for the problem at hand. Comparison with results obtained for a massive scalar field in $\mathbb{S}^{3}$ and final remarks are left for the last Section. Throughout this paper we employ natural units, $\hbar=c=1$; Boltzmann constant $k_{B}$ is also set equal to the unity.

\section{QUANTUM DYNAMICS OF EXTERIOR FORMS OF REAL HYPERBOLIC SPACES}

Let $X_{\Gamma}$ be a $N$-dimensional real compact hyperbolic space with universal covering $M$ and fundamental group 
$\Gamma$. Then we can represent $M$ as the symmetric space $G / K$, where $G=S O_{1}(N, 1)$ and $K=S O(N)$ is a maximal compact subgroup of $G$. We regard $\Gamma$ as a discrete subgroup of $G$ acting isometrically on $M$, and we take $X_{\Gamma}$ to be the quotient space by that action: $X_{\Gamma}=\Gamma \backslash M=\Gamma \backslash G / K$. Let $\tau$ be an irreducible representation of $K$ on a complex vector space $V_{\tau}$, and form the induced homogeneous vector bundle $G \times{ }_{K} V_{\tau}$ (the fiber product of $G$ with $V_{\tau}$ over $K$ ) over $M$. Restricting the $G$ action to $\Gamma$ we obtain the quotient bundle $E_{\tau}=\Gamma \backslash\left(G \times_{K} V_{\tau}\right) \rightarrow X_{\Gamma}$. The natural Riemannian structure on $M$ (therefore on $X_{\Gamma}$ ) induced by the Killing form (, ) of $G$ gives rise to a connection Laplacian $L$ on $E_{\tau}$. If $\Omega_{K}$ denotes the Casimir operator of $K$ - that is $\Omega_{K}=-\sum y_{j}^{2}$, for a basis $\left\{y_{j}\right\}$ of the Lie algebra $\mathfrak{k}_{0}$ of $K$, where $\left(y_{j}, y_{\ell}\right)=-\delta_{j \ell}$, then $\tau\left(\Omega_{K}\right)=\lambda_{\tau}$ for a suitable scalar $\lambda_{\tau}$. Moreover, for the Casimir operator $\Omega$ of $G$, with $\Omega$ operating on smooth sections $\Gamma^{\infty} E_{\tau}$ of $E_{\tau}$ we have

$$
L=\Omega-\lambda_{\tau} \mathbf{1}
$$

see Lemma 3.1 of Ref. [5]. For $\lambda \geq 0$ let

$$
\Gamma^{\infty}\left(X, E_{\tau}\right)_{\lambda}=\left\{s \in \Gamma^{\infty} E_{\tau} \mid-L s=\lambda s\right\}
$$

be the space of eigensections of $L$ corresponding to $\lambda$. Here we note that since $X_{\Gamma}$ is compact we can order the spectrum of $-L$ by taking $0=\lambda_{0}<\lambda_{1}<\lambda_{2}<\cdots$; $\lim _{j \rightarrow \infty} \lambda_{j}=\infty$. We shall focus on the case when $N=2 k$ is even, and we shall specialize $\tau$ to be the representation $\tau_{p}$ of $K=S O(2 k)$ on $\Lambda^{p} \mathbb{C}^{2 k}$, say $p \neq k$. The case when $N$ is odd will be dealt with later. It will be convenient moreover to work with the normalized Laplacian $\mathfrak{L}=$ $-c(N) L$ where $c(N)=2(N-1)=2(2 k-1) . \quad \mathfrak{L}$ has spectrum $\left\{c(N) \lambda_{j}, m_{j}\right\}_{j=0}^{\infty}$ where the multiplicity $m_{j}$ of the eigenvalue $c(N) \lambda_{j}$ is given by

$$
m_{j}=\operatorname{dim} \Gamma^{\infty}\left(X, E_{\tau_{p}}\right)_{\lambda_{j}} .
$$

Let $\omega_{p}, \varphi_{p}$ be exterior differential $p$-forms; then, the invariant inner product is defined by $\left(\omega_{p}, \varphi_{p}\right) \stackrel{\text { def }}{=} \int_{X_{\Gamma}} \omega_{p} \wedge$ $* \varphi_{p}$. The following properties for operators and forms hold: $d d=\delta \delta=0, \delta=(-1)^{N p+N+1} * d *, * * \omega_{p}=$ $(-1)^{p(N-p)} \omega_{p}$. The operators $d$ and $\delta$ are adjoint to each other with respect to this inner product for $p$-forms: $\left(\delta \omega_{p}, \varphi_{p}\right)=\left(\omega_{p}, d \varphi_{p}\right)$. In quantum field theory the Lagrangian associated with $\omega_{p}$ takes the form: $d \omega_{p} \wedge * d \omega_{p}$ (gauge field), and $\delta \omega_{p} \wedge * \delta \omega_{p}$ (co-gauge field). The Euler-Lagrange equations, supplied with the gauge, give: $\mathfrak{L}_{p} \omega_{p}=0, \delta \omega_{p}=0$ (Lorentz gauge); $\mathfrak{L}_{p} \omega_{p}=0, d \omega_{p}=0$ (co-Lorentz gauge). These Lagrangians provide a possible representation of tensor fields or generalized abelian gauge fields. The two representations of tensor fields are not completely independent, because of the wellknown duality property of exterior calculus which gives a connection between star-conjugated gauge and co-gauge tensor fields. The gauge $p$-forms are mapped into the co-gauge $(N-p)$-forms under the action of the Hodge
* operator. The vacuum-to-vacuum amplitude for the gauge $p-$ form $\omega_{p}$ becomes $[\underline{6}]$ :

$$
\begin{aligned}
Z & =\mathcal{N} \int D \omega \exp \left[-\left(\omega, \mathfrak{L}_{p} \omega\right)\right] \\
& \times \prod_{j=1}^{p}\left(\operatorname{Vol}_{p-j}\left(\operatorname{det} \mathfrak{L}_{p-j}\right)^{(j+1) / 2}\right)^{(-1)^{j+1}}
\end{aligned}
$$

where we need to factorize the divergent gauge group volume and integrate over the classes of gauge transformations $(\omega \rightarrow \omega+d \phi)$.

\section{THE TRACE FORMULA APPLIED TO THE TENSOR KERNEL}

The space of smooth sections $\Gamma^{\infty} E_{\tau}$ of $E_{\tau}$ is just the space of smooth $p$-forms on $X$. We can therefore apply the version of the trace formula developed by Fried in [7]. First we set up some additional notation. For $\sigma_{p}$ the natural representation of $S O(2 k-1)$ on $\Lambda^{p} \mathbb{C}^{2 k-1}$, we have the corresponding Harish-Chandra-Plancherel density given - for a suitable normalization of the Haar measure $d x$ on $G$ - by

$$
\mu_{\sigma_{p}(r)}=\frac{\pi}{2^{4 k-4}[\Gamma(k)]^{2}}\left(\begin{array}{c}
2 k-1 \\
p
\end{array}\right) P_{\sigma_{p}}(r) r \tanh (\pi r),
$$

for $0 \leq p \leq k-1$, where

$$
\begin{aligned}
P_{\sigma_{p}}(r) & =\prod_{\ell=2}^{p+1}\left[r^{2}+\left(k-\ell+\frac{3}{2}\right)^{2}\right] \\
& \times \prod_{\ell=p+2}^{k}\left[r^{2}+\left(k-\ell+\frac{1}{2}\right)^{2}\right]
\end{aligned}
$$

is an even polynomial of degree $2 k-2$. We have that $P_{\sigma_{p}}(r)=P_{\sigma_{2 k-1-p}}(r)$ and $\mu_{\sigma_{p}}(r)=\mu_{\sigma_{2 k-1-p}}(r)$ for $k \leq$ $p \leq 2 k-1$. Define the Miatello coefficients $[8] a_{2 \ell}^{(p)}$ for $G=S O_{1}(2 k+1,1)$ by

$$
P_{\sigma_{p}}(r)=\sum_{\ell=0}^{k-1} a_{2 \ell}^{(p)} r^{2 \ell}, \quad 0 \leq p \leq 2 k-1 .
$$

Let $\operatorname{Vol}(\Gamma \backslash G)$ will denote the integral of the constant function 1 on $\Gamma \backslash G$ with respect to the $G$ - invariant measure on $\Gamma \backslash G$ induced by $d x$. For $0 \leq p \leq N-1$ the Fried trace formula applied to kernel holds [7]:

$$
\begin{aligned}
\operatorname{Tr}\left(e^{-t \mathfrak{L}_{p}}\right) & =I_{\Gamma}^{(p)}\left(\mathcal{K}_{t}\right)+I_{\Gamma}^{(p-1)}\left(\mathcal{K}_{t}\right) \\
& +H_{\Gamma}^{(p)}\left(\mathcal{K}_{t}\right)+H_{\Gamma}^{(p-1)}\left(\mathcal{K}_{t}\right),
\end{aligned}
$$

where $I_{\Gamma}^{(p)}\left(\mathcal{K}_{t}\right), H_{\Gamma}^{(p)}\left(\mathcal{K}_{t}\right)$ are the identity and hyperbolic orbital integral respectively. In the above formula

$$
\begin{aligned}
I_{\Gamma}^{(p)}\left(\mathcal{K}_{t}\right) \stackrel{\text { def }}{=} & \frac{\chi(1) \operatorname{Vol}(\Gamma \backslash G)}{4 \pi} \int_{\mathbb{R}} d r \mu_{\sigma_{p}}(r) \\
& \times e^{-t\left(r^{2}+b^{(p)}+\left(\rho_{0}-p\right)^{2}\right)},
\end{aligned}
$$




$$
\begin{aligned}
H_{\Gamma}^{(p)}\left(\mathcal{K}_{t}\right) \stackrel{\text { def }}{=} & \frac{1}{\sqrt{4 \pi t}} \sum_{\gamma \in C_{\Gamma}-\{1\}} \frac{\chi(\gamma)}{j(\gamma)} t_{\gamma} C(\gamma) \chi_{\sigma_{p}}\left(m_{\gamma}\right) \\
& \times e^{-t\left(b^{(p)}+\left(\rho_{0}-p\right)^{2}\right)-t_{\gamma}^{2} / 4 t}
\end{aligned}
$$

where $C_{\Gamma} \subset \Gamma$ is a complet set of representations in $\Gamma$ of its conjugacy classes, $C(\gamma)$ is a well defined function on $\Gamma-\{1\}$ (for more details see Ref. [9]), $\rho_{0}=(N-1) / 2$, $b^{(p)}$ are real constants, and $\chi_{\sigma}(m)=\operatorname{trace}(\sigma(m))$ is the character $\sigma$ for $m \in S O(2 k-1)$.

For $p \geq 1$ there is a measure $\mu_{\sigma}(r)$ corresponding to a general irreducible representation $\sigma$. Let $\sigma_{p}$ be the standard representation of $S O(N-1)$ on $\Lambda^{p} \mathbb{C}^{(N-1)}$. If $N=2 k$ is even then $\sigma_{p}(0 \leq p \leq N-1)$ is always irreducible; if $N=2 k+1$ then every $\sigma_{p}$ is irreducible except for $p=(N-1) / 2=k$, in which case $\sigma_{k}$ is the direct sum of two spin-(1/2) representations $\sigma^{ \pm}: \sigma_{k}=\sigma^{+} \oplus \sigma^{-}$. For $p=k$ the representation $\tau_{k}$ of $K=S O(2 k)$ on $\Lambda^{k} \mathbb{C}^{2 k}$ is not irreducible: $\tau_{k}=\tau_{k}^{+} \oplus \tau_{k}^{-}$is the direct sum of two spin-(1/2) representations. In the case of the trivial representation ( $p=0$, i.e. for smooth functions or smooth vector bundle sections) the measure $\mu(r) \equiv \mu_{0}(r)$ corresponds to the trivial representation. Therefore, we take $I_{\Gamma}^{(-1)}\left(\mathcal{K}_{t}\right)=H_{\Gamma}^{(-1)}\left(\mathcal{K}_{t}\right)=0$. Since $\sigma_{0}$ is the trivial representation, we have $\chi_{\sigma_{0}}\left(m_{\gamma}\right)=1$. In this case, formula (8) reduces exactly to the trace formula for $p=0$ [5, 9, 10, 11],

$$
\begin{aligned}
I_{\Gamma}^{(0)}\left(\mathcal{K}_{t}\right) & =\frac{\chi(1) \operatorname{vol}(\Gamma \backslash G)}{4 \pi} \int_{\mathbb{R}} d r \mu_{\sigma_{0}}(r) e^{-t\left(r^{2}+b^{(0)}+\rho_{0}^{2}\right)}, \\
H_{\Gamma}^{(0)}\left(\mathcal{K}_{t}\right) & =\frac{1}{\sqrt{4 \pi t}} \sum_{\gamma \in C_{\Gamma}-\{1\}} \frac{\chi(\gamma)}{j(\gamma)} t_{\gamma} C(\gamma) e^{-t\left(b^{(0)}+\rho_{0}^{2}\right)-\frac{t_{\gamma}^{2}}{4 t}} .
\end{aligned}
$$

\section{THE SPECTRAL FUNCTIONS OF EXTERIOR FORMS}

The spectral zeta function related to the Laplace operator $\mathfrak{L}_{j}$ can be represented by the inverse Mellin transform of the heat kernel $\mathcal{K}_{t}=\operatorname{Tr} \exp \left(-t \mathfrak{L}_{j}\right)$. Using the Fried formula, we can write the zeta function as a sum of contributions:

$$
\begin{aligned}
& \zeta\left(s \mid \mathfrak{L}_{j}\right)=\frac{1}{\Gamma(s)} \int_{0}^{\infty} d t t^{s-1} \\
& \times\left(I_{\Gamma}^{(j)}\left(\mathcal{K}_{t}\right)+I_{\Gamma}^{(j-1)}\left(\mathcal{K}_{t}\right)+H_{\Gamma}^{(j)}\left(\mathcal{K}_{t}\right)+H_{\Gamma}^{(j-1)}\left(\mathcal{K}_{t}\right)\right) \\
& \equiv \zeta_{I}^{(N)}(s, j)+\zeta_{I}^{(N)}(s, j-1) \\
& +\zeta_{H}^{(N)}(s, j)+\zeta_{H}^{(N)}(s, j-1)
\end{aligned}
$$

For the identity component we have

$$
\zeta_{I}^{(N)}(s, j)=\frac{V_{\Gamma}}{\Gamma(s)} \int_{0}^{\infty} d t t^{s-1} \int_{\mathbb{R}} d r \mu_{\sigma_{j}} e^{-t\left(r^{2}+\alpha_{j}^{2}\right)},
$$

where $V_{\Gamma}=\chi(1) \operatorname{Vol}(\Gamma \backslash G) / 4 \pi$, and we define $\alpha_{j}^{2}=b^{(j)}+$ $\left(\rho_{0}-j\right)^{2}$. Replacing the Harish-Chandra-Plancherel measure, we obtain two representations for $\zeta_{I}^{(N)}(s, j)$, which holds for the cases of odd and even dimension. Thus,

$$
\begin{aligned}
& \zeta_{I}^{(2 k)}(s, j)=\frac{V_{\Gamma} C_{2 k}^{(j)}}{\Gamma(s)} \sum_{\ell=0}^{k-1} a_{2 \ell, 2 k}^{(j)} \\
& \times \int_{0}^{\infty} d t t^{s-1} \int_{\mathbb{R}} d r r^{2 \ell+1} \tanh (\pi r) e^{-t\left(r^{2}+\alpha_{j}^{2}\right)} .
\end{aligned}
$$

Using the identities

$$
\begin{gathered}
\tanh (\pi r)=1-\frac{2}{1+e^{2 \pi r}}, \\
\int_{0}^{\infty} \frac{d r r^{2 \ell-1}}{1+e^{2 \pi r}}=(-1)^{\ell-1} \frac{\left(1-2^{1-2 \ell}\right) B_{2 \ell}}{4 \ell},
\end{gathered}
$$

where $B_{\ell}$ is the $\ell$-th Bernoulli number, we get

$$
\begin{aligned}
& \zeta_{I}^{(2 k)}(s, j)=\frac{V_{\Gamma} C_{2 k}^{(j)}}{\Gamma(s)} \sum_{\ell=0}^{k-1} a_{2 \ell, 2 k}^{(j)} \\
& \times\left[\Gamma(\ell+1) \Gamma(s-\ell-1) \alpha_{j}^{-2 s+2 \ell+2}\right. \\
& \left.+\sum_{n=0}^{\infty} \xi_{n \ell} \Gamma(s+n) \alpha_{j}^{-2 s-2 n}\right]
\end{aligned}
$$

where we have defined

$$
\xi_{n \ell} \stackrel{\text { def }}{=} \frac{(-1)^{\ell+1}\left(1-2^{-2 \ell-2 n-1}\right)}{n !(2 \ell+2 n+2)} B_{2 \ell+2 n+2} .
$$

In the odd dimensional case we get

$$
\begin{aligned}
& \zeta_{I}^{(2 k+1)}(s, j)=\frac{V_{\Gamma} C_{2 k+1}^{(j)}}{\Gamma(s)} \sum_{\ell=0}^{k} a_{2 \ell, 2 k+1}^{(j)} \\
& \times \int_{0}^{\infty} d t t^{s-1} \int_{\mathbb{R}} d r r^{2 \ell} e^{-t\left(r^{2}+\alpha_{j}^{2}\right)} \\
& =\frac{V_{\Gamma} C_{2 k+1}^{(j)}}{\Gamma(s)} \sum_{\ell=0}^{k} a_{2 \ell, 2 k+1}^{(j)} \Gamma\left(\ell+\frac{1}{2}\right) \\
& \times \Gamma\left(s-\ell-\frac{1}{2}\right) \alpha_{j}^{-2 s+2 \ell+1} .
\end{aligned}
$$

The hyperbolic component of the zeta function takes the form

$$
\zeta_{H}^{(N)}(s, j)=\sum_{\gamma \in C_{\Gamma}-\{1\}} \frac{\chi(\gamma) t_{\gamma} C(\gamma) \chi_{\sigma_{j}}\left(m_{\gamma}\right)}{\sqrt{4 \pi} \Gamma(s) j(\gamma)} \int_{0}^{\infty} d t \frac{e^{-t \alpha_{j}^{2}-\frac{t_{\gamma}^{2}}{4 t}}}{t^{-s+\frac{3}{2}}}
$$

Using the McDonald function,

$$
K_{\nu}(z)=\frac{1}{2}\left(\frac{z}{2}\right)^{\nu} \int_{0}^{\infty} d t \frac{e^{-t-\frac{z^{2}}{4 t}}}{t^{\nu+1}}
$$


where $|\arg z|<\pi / 2$ and $\Re z^{2}>0$, we obtain

$$
\zeta_{H}^{(N)}(s, j)=\sum_{\gamma \in C_{\Gamma}-\{1\}} \frac{\chi(\gamma) t_{\gamma}^{2 s} C(\gamma) \chi_{\sigma_{j}}\left(m_{\gamma}\right)}{\sqrt{\pi} \Gamma(s) j(\gamma)} \frac{K_{-s+\frac{1}{2}}\left(\alpha_{j} t_{\gamma}\right)}{\left(2 \alpha t_{\gamma}\right)^{s-1 / 2}} .
$$

\section{THE HIGH TEMPERATURE EXPANSIONS}

Using the Mellin representation for the zeta function, we can obtain useful formulas for the temperature dependent part of the identity and hyperbolic orbital components of the free energy (see for detail Refs. 10, 11, 12])

$F_{I, H}^{(N)}(\beta, j)=-\frac{1}{2 \pi i} \int_{\Re z=c} \frac{d z}{\beta^{z}} \zeta(z) \Gamma(z-1) \zeta_{I, H}\left(\frac{z-1}{2}, j\right)$,

where $\zeta(z)$ is the Riemann zeta function. A tedious calculation gives the following results:

$$
\begin{aligned}
F_{I}^{(2 k)}(\beta, j) & =-\frac{V_{\Gamma} C_{2 k}^{(j)} a_{2 k-2,2 k}^{(j)}}{\sqrt{4 \pi}} \Gamma(k) \zeta(2 k+1) \\
& \times \Gamma\left(k+\frac{1}{2}\right) \beta^{-2 k-1} \\
& -\frac{V_{\Gamma} C_{2 k}^{(j)}}{\sqrt{4 \pi}} \zeta(2 k-1) \Gamma\left(k-\frac{1}{2}\right) \\
& \times\left[a_{2 k-4,2 k}^{(j)} \Gamma(k-1)\right. \\
& \left.-a_{2 k-2,2 k}^{(j)} \Gamma(k)\right] \beta^{-2 k+1} \\
& +\mathcal{O}\left(\beta^{-2 k+3}\right), \\
F_{I}^{(2 k+1)}(\beta, j) & =-\frac{V_{\Gamma} C_{2 k+1}^{(j)} a_{2 k, 2 k+1}^{(j)}}{\sqrt{4 \pi}} \Gamma\left(k+\frac{1}{2}\right) \\
& \times \zeta(2 k+2) \Gamma(k+1) \beta^{-2 k-2} \\
& -\frac{V_{\Gamma} C_{2 k+1}^{(j)}}{\sqrt{4 \pi}} \zeta(2 k) \Gamma(k) \\
& \times\left[a_{2 k-2,2 k+1}^{(j)} \Gamma\left(k-\frac{1}{2}\right)\right. \\
& \left.-a_{2 k, 2 k+1}^{(j)} \Gamma\left(k+\frac{1}{2}\right) \alpha_{j}^{2}\right] \beta^{-2 k} \\
& +\mathcal{O}\left(\beta^{-2 k+2}\right) .
\end{aligned}
$$

Note that the contribution associated to the hyperbolic orbital component is negligible small.

\section{A. The thermodynamic functions and the entropy bound}

In the context of the Hodge theory, the physical degrees of freedom are represented by the co-exact part of the $p$-form. For $0 \leq p \leq N-1$ the Fried trace formula [7] applied to the tensor kernel associated with co-exact forms has to be modified [13, 14, 15]:

$$
\begin{aligned}
& \operatorname{Tr}\left(e^{-t \mathfrak{L}_{p}^{(C E)}}\right)=\sum_{j=1}^{p}(-1)^{j}\left[I_{\Gamma}^{(p-j)}\left(\mathcal{K}_{t}\right)+I_{\Gamma}^{(p-1-j)}\left(\mathcal{K}_{t}\right)\right. \\
& \left.+H_{\Gamma}^{(p-j)}\left(\mathcal{K}_{t}\right)+H_{\Gamma}^{(p-1-j)}\left(\mathcal{K}_{t}\right)-b_{p-j}\right],
\end{aligned}
$$

where $b_{j}$ are the Betti numbers. Thus, the free energy becomes

$$
\begin{aligned}
\mathcal{F}^{(N)}(\beta) & =\sum_{j=0}^{p}(-1)^{j}\left(F_{I}^{(N)}(\beta, p-j)\right. \\
& \left.+F_{I}^{(N)}(\beta, p-j-1)\right) .
\end{aligned}
$$

In the high temperature limit $(\beta \rightarrow 0)$ we have

$$
\begin{aligned}
\mathcal{F}^{(N)}(\beta) & =-A_{1}(N ; \Gamma) \beta^{-N-1} \\
& -A_{2}(N ; \Gamma) \beta^{-N+1}+\mathcal{O}\left(\beta^{-N+3}\right),
\end{aligned}
$$

where for the even dimensional case,

$$
\begin{aligned}
A_{1}(2 k ; \Gamma) & =\frac{V_{\Gamma}}{\sqrt{4 \pi}} \zeta(2 k+1) \Gamma(k) \Gamma\left(k+\frac{1}{2}\right) \\
& \times C_{2 k}^{(p)} a_{2 k-2,2 k}^{(p)}, \\
A_{2}(2 k ; \Gamma)= & \frac{V_{\Gamma}}{\sqrt{4 \pi}} \zeta(2 k-1) \Gamma(k-1) \Gamma\left(k-\frac{1}{2}\right) \\
& \times C_{2 k}^{(p)}\left(a_{2 k-4,2 k}^{(p)}+(k-1) a_{2 k-2,2 k}^{(p)}\right),
\end{aligned}
$$

and for the odd dimensional case,

$$
\begin{gathered}
A_{1}(2 k+1 ; \Gamma)=\frac{V_{\Gamma}}{\sqrt{4 \pi}} \zeta(2 k+2) \Gamma\left(k+\frac{1}{2}\right) \Gamma(k+1) \\
\times C_{2 k+1}^{(p)} a_{2 k, 2 k+1}^{(p)} \\
A_{2}(2 k+1 ; \Gamma)=\frac{V_{\Gamma}}{\sqrt{4 \pi}} \zeta(2 k) \Gamma\left(k-\frac{1}{2}\right) \Gamma(k) \\
\times C_{2 k+1}^{(p)}\left(a_{2 k-2,2 k+1}^{(p)}-\left(k-\frac{1}{2}\right) a_{2 k, 2 k+1}^{(p)} \alpha_{p}^{2}\right)
\end{gathered}
$$

The entropy and the total energy can be obtained by means of the following thermodynamic relations: $S^{(N)}(\beta)=\beta^{2} \partial \mathcal{F}^{(N)}(\beta) / \partial \beta, \quad E^{(N)}(\beta)=$ $\partial\left(\beta \mathcal{F}^{(N)}(\beta)\right) / \partial \beta$. Therefore,

$$
\begin{aligned}
S^{(N)}(\beta)= & (N+1) A_{1}(N ; \Gamma) \beta^{-N} \\
& +(N-1) A_{2}(N ; \Gamma) \beta^{-N+2} \\
& +\mathcal{O}\left(\beta^{-N+4}\right), \\
E^{(N)}(\beta)= & -N A_{1}(N ; \Gamma) \beta^{-N-1} \\
& -(N-2) A_{2}(N ; \Gamma) \beta^{-N+1} \\
& +\mathcal{O}\left(\beta^{-N+3}\right),
\end{aligned}
$$


The ratio entropy/energy becomes

$$
\frac{S^{(N)}(\beta)}{E^{(N)}(\beta)}=\frac{N+1}{N} \beta+\frac{2}{N^{2}} \frac{A_{2}(N ; \Gamma)}{A_{1}(N ; \Gamma)} \beta^{3}+\mathcal{O}\left(\beta^{5}\right) .
$$

\section{THE LOW TEMPERATURE EXPANSIONS}

In the low temperature limit we can use the following representation for the one-loop contribution to the free energy [10, 11]:

$$
\mathcal{F}^{(N)}(\beta)=-\frac{1}{\sqrt{\pi}} \sum_{n=1}^{\infty} \int_{0}^{\infty} d t t^{-3 / 2} e^{-n^{2} \beta^{2} / 4 t} \operatorname{Tr} e^{-t \mathfrak{L}_{p}^{(C E)}}
$$

For the identity contribution we have

$$
\begin{aligned}
F_{I}^{(N)}(\beta, j)= & \frac{V_{\Gamma}}{\sqrt{\pi}} \sum_{n=1}^{\infty} \int_{0}^{\infty} d t t^{-3 / 2} \int_{\mathbb{R}} d r \mu_{\sigma_{j}}(r) \\
& \times e^{-t\left(\alpha_{j}^{2}+r^{2}\right)-n^{2} \beta^{2} / 4 t} .
\end{aligned}
$$

Therefore, the following formulas hold

$$
\begin{aligned}
& F_{I}^{(2 k)}(\beta, j)=\frac{2 V_{\Gamma} C_{N}^{(j)}}{\sqrt{\pi}} \sum_{n=1}^{\infty} \sum_{\ell=0}^{k-1} a_{2 \ell, 2 k}^{(j)}\left[2^{3 / 2} \Gamma(\ell+1)\right. \\
& \times \alpha_{j}^{2 \ell+3} \frac{K_{\ell+\frac{3}{2}}\left(\alpha_{j} n \beta\right)}{\left(\alpha_{j} n \beta\right)^{\ell+3 / 2}} \\
& -(-1)^{\ell} \sum_{m=0}^{\infty} \frac{\left(1-2^{-2 \ell-2 m-1}\right) B_{2 \ell+2 m+2}}{m !(\ell+m+1) 2^{m-1 / 2}} \\
& \left.\times \alpha_{j}^{-2 m+1} \frac{K_{-m+\frac{1}{2}}\left(\alpha_{j} n \beta\right)}{\left(\alpha_{j} n \beta\right)^{-m+1 / 2}}\right] \text {, } \\
& F_{I}^{(2 k+1)}(\beta, j)=\frac{2 V_{\Gamma} C_{N}^{(j)}}{\sqrt{\pi}} \sum_{n=1}^{\infty} \sum_{\ell=0}^{k} a_{2 \ell, 2 k+1}^{(j)} \Gamma\left(\ell+\frac{1}{2}\right) \\
& \times\left(2 \alpha_{j}^{2}\right)^{\ell+1} \frac{K_{\ell+1}\left(\alpha_{j} n \beta\right)}{\left(\alpha_{j} n \beta\right)^{\ell+1}} \\
& F_{H}^{(N)}(\beta, j)=2 \alpha_{j}^{2} \sum_{n=1}^{\infty} \sum_{\gamma \in C_{\Gamma}-\{1\}} \frac{\chi(\gamma) t_{\gamma} C(\gamma) \chi_{\sigma_{p}}\left(m_{\gamma}\right)}{\pi j(\gamma)} \\
& \times \frac{K_{1}\left(\alpha_{N} \sqrt{n^{2} \beta^{2}+t_{\gamma}^{2}}\right)}{\left(\alpha_{N} \sqrt{n^{2} \beta^{2}+t_{\gamma}^{2}}\right)} .
\end{aligned}
$$

Using the asymptotic expansion for the McDonald function (22)

$$
\begin{aligned}
K_{\nu}(z)= & \sqrt{\frac{\pi}{2 z}} e^{-z}\left[\sum_{k=0}^{\ell-1} \frac{\Gamma(\nu+k+1 / 2)}{(2 z)^{k} k ! \Gamma(\nu-k-1 / 2)}\right. \\
& \left.+\frac{\theta \Gamma(\nu+\ell+1 / 2)}{(2 z)^{\ell} \ell ! \Gamma(\nu-\ell+1 / 2)}\right]
\end{aligned}
$$

for $\nu \in \mathbb{R}, z>0, \ell>\nu-1 / 2(\ell=1,2,3, \ldots),|\theta| \leq 1$ (see [16], page 963), in Eqs. (39), (40) and (41) we get the following result for the entropy

$$
\begin{aligned}
S^{(N)}(\beta)= & \widetilde{A}_{1}(N ; \Gamma) \beta^{1 / 2}+\widetilde{A}_{2}(N ; \Gamma) \beta^{-1 / 2} \\
& +\mathcal{O}\left(\beta^{-3 / 2}\right)
\end{aligned}
$$

where

$$
\begin{aligned}
& \widetilde{A}_{1}(2 k ; \Gamma)=\sum_{\gamma \in C_{\Gamma}-\{1\}} \frac{2 \chi(\gamma) t_{\gamma} C(\gamma)}{\sqrt{2 \pi} j(\gamma)} \chi_{\sigma_{p}}\left(m_{\gamma}\right) \alpha_{p}^{3 / 2} \\
& -\frac{V_{\Gamma}}{2 \pi} \sum_{\ell=0}^{k-1} \frac{(-1)^{\ell}\left(1-2^{-2 \ell-1}\right) B_{2 \ell+2}}{\ell+1} \\
& \times C_{2 k}^{(p)} a_{2 \ell, 2 k}^{(p)} \alpha_{p}^{1 / 2}, \\
& \widetilde{A}_{2}(2 k ; \Gamma)=\frac{V_{\Gamma}}{\pi} C_{2 k}^{(p)} a_{0,2 k}^{(p)} \alpha_{p}^{3 / 2} \\
& +\sum_{\gamma \in C_{\Gamma}-\{1\}} \frac{15 \chi(\gamma) t_{\gamma} C(\gamma)}{8 \sqrt{2 \pi} J(\gamma)} \chi_{\sigma_{p}}\left(m_{\gamma}\right) \alpha_{p}^{1 / 2} \\
& +\frac{3 V_{\Gamma}}{4 \pi} \sum_{\ell=0}^{k-1} \frac{(-1)^{\ell}\left(1-2^{-2 \ell-1}\right) B_{2 \ell+2}}{\ell+1} \\
& \times C_{2 k}^{(p)} a_{2 \ell, 2 k}^{(p)} \alpha_{p}^{-1 / 2}, \\
& \widetilde{A}_{1}(2 k+1 ; \Gamma)=\sum_{\gamma \in C_{\Gamma}-\{1\}} \frac{2 \chi(\gamma) t_{\gamma} C(\gamma)}{\sqrt{2 \pi} j(\gamma)} \chi_{\sigma_{p}}\left(m_{\gamma}\right) \alpha_{p}^{3 / 2} \\
& +\frac{V_{\Gamma}}{\sqrt{2 \pi}} C_{2 k+1}^{(p)} a_{0,2 k+1}^{(p)} \alpha_{p}^{3 / 2}, \\
& \widetilde{A}_{2}(2 k+1 ; \Gamma)=\sum_{\gamma \in C_{\Gamma}-\{1\}} \frac{\chi(\gamma) t_{\gamma} C(\gamma)}{\sqrt{2 \pi} j(\gamma)} \chi_{\sigma_{p}}\left(m_{\gamma}\right) \\
& \times\left(3 \alpha_{p}^{1 / 2}+2 \alpha_{p}^{3 / 2}\right) \\
& +\frac{15 V_{\Gamma}}{8 \sqrt{2 \pi}} C_{2 k+1}^{(p)} a_{0,2 k+1}^{(p)} \alpha_{p}^{1 / 2} .
\end{aligned}
$$

The energy is given by

$$
\begin{aligned}
E^{(N)}(\beta) & =-\widetilde{A}_{1}(N ; \Gamma) \beta^{-1 / 2}-\widetilde{A}_{3}(N ; \Gamma) \beta^{-3 / 2} \\
& +\mathcal{O}\left(\beta^{-5 / 2}\right)
\end{aligned}
$$

where

$$
\begin{aligned}
\widetilde{A}_{3}(2 k ; \Gamma) & =-\frac{V_{\Gamma}}{\pi} C_{2 k}^{(p)} a_{0,2 k}^{(p)} \alpha_{p}^{3 / 2} \\
& -\frac{7}{4} \sum_{\gamma \in C_{\Gamma}-\{1\}} \frac{\chi(\gamma) t_{\gamma} C(\gamma)}{\sqrt{2 \pi} J(\gamma)} \chi_{\sigma_{p}}\left(m_{\gamma}\right) \alpha_{p}^{1 / 2} \\
& +\frac{V_{\Gamma}}{4 \pi} \sum_{\ell=0}^{k-1} \frac{(-1)^{\ell}\left(1-2^{-2 \ell-1}\right) B_{2 \ell+2}}{\ell+1} \\
& \times C_{2 k}^{(p)} a_{2 \ell, 2 k}^{(p)} \alpha_{p}^{-1 / 2}
\end{aligned}
$$




$$
\begin{aligned}
\widetilde{A}_{3}(2 k+1 ; \Gamma) & =\sum_{\gamma \in C_{\Gamma}-\{1\}} \frac{\chi(\gamma) t_{\gamma} C(\gamma)}{\sqrt{2 \pi} j(\gamma)} \chi_{\sigma_{p}}\left(m_{\gamma}\right) \\
& \times\left(\alpha_{p}^{1 / 2}+2 \alpha_{p}^{3 / 2}\right) \\
& +\frac{7 V_{\Gamma}}{8 \sqrt{2 \pi}} C_{2 k+1}^{(p)} a_{0,2 k+1}^{(p)} \alpha_{p}^{1 / 2}
\end{aligned}
$$

For the entropy/energy ratio in low temperature limit we get

$$
\begin{aligned}
\frac{S^{(N)}(\beta)}{E^{(N)}(\beta)} & =\beta-\frac{\widetilde{A}_{3}(N ; \Gamma)}{\widetilde{A}_{2}(N ; \Gamma)}\left(\frac{\widetilde{A}_{2}(N ; \Gamma)}{\widetilde{A}_{1}(N ; \Gamma)}-\frac{\widetilde{A}_{3}(N ; \Gamma}{\widetilde{A}_{1}(N ; \Gamma)}\right) \beta^{-1} \\
& +\mathcal{O}\left(\beta^{-2}\right)
\end{aligned}
$$

\section{CONCLUSIONS}

We have considered gauge theories based on abelian $p$-forms in real compact hyperbolic manifolds. The explicit thermodynamic functions associated with skewsymmetric tensor fields are obtained by using zetafunction regularization and the trace tensor kernel formula. Thermodynamic quantities in the low and high temperature limits were calculated. We also have obtained the entropy/energy ratios (in both temperature limits). The dependence on the Miatello coefficients related to the structure of the Harish-Chandra-Plancherel measure stems from the second term of the expansion. In the case of scalar fields $(p=0)$ we have Eq. (36) with

$$
\begin{aligned}
\frac{A_{2}(2 k ; \Gamma)}{A_{1}(2 k ; \Gamma)} & =\frac{2}{2 k-1} \frac{\zeta(2 k-1)}{\zeta(2 k+1)} \\
& \times\left(\frac{1}{k-1} \frac{a_{2 k-4,2 k}^{(0)}}{a_{2 k-2,2 k}^{(0)}}-\alpha_{0}^{2}\right),
\end{aligned}
$$

$$
\begin{aligned}
\frac{A_{2}(2 k+1 ; \Gamma)}{A_{1}(2 k+1 ; \Gamma)} & =\frac{1}{k} \frac{\zeta(2 k)}{\zeta(2 k+2)} \\
& \times\left(\frac{2}{2 k-1} \frac{a_{2 k-2,2 k+1}^{(0)}}{a_{2 k, 2 k+1}^{(0)}}-\alpha_{0}^{2}\right),
\end{aligned}
$$

where $\alpha_{0}^{2}=\rho_{0}^{2}+m^{2}\left(\alpha_{0}^{2}=\rho_{0}^{2}\right.$ for the massless case). For three-dimensional hyperbolic manifolds the Miatello coefficients read [17]: $a_{0}^{(0)}=a_{2}^{(0)}=1$ and therefore

$$
\frac{S^{(3)}(\beta)}{E^{(3)}(\beta)}=\frac{4}{3} \beta+\frac{10}{3 \pi^{2}}\left(2-\alpha_{0}^{2}\right) \beta^{3}+\mathcal{O}\left(\beta^{5}\right)
$$

This formula is in agreement with the result obtained in [4] where entropy bounds were calculated for spherical geometry and where the dependence on geometry of the backgrounds also stems from the second term of the expansion.

\section{Acknowledgements}

A.A.B. would like to thank Fundação de Amparo à Pesquisa do Estado de São Paulo (FAPESP/Brazil) and the Conselho Nacional de Desenvolvimento Científico e Tecnológico (CNPq/Brazil) for partial financial support, and the Instituto de Física Teórica (IFT/UNESP) for kind hospitality. V.S.M. thanks CAPES for PhD grant.
[1] N. D. Birrel and P. C. W. Davies, Quantum Fields in Curved Spacetime (Cambridge University Press, 1982).

[2] L. H. Ford, Phys. Rev. D 11, 535 (1975).

[3] I. Brevik, K. A. Milton and S. D. Odintsov, Ann. of Phys. 302, 120 (2002).

[4] E. Elizalde and A. C. Tort, Phys. Rev. D 67, 124014 (2003).

[5] N. Wallach, J. Diff. Geom. 11, 91 (1976).

[6] Yu. N. Obukhov, Phys. Lett. B 109, 195 (1982).

[7] D. Fried, Invent. Math. 84, 523 (1986).

[8] R. Miatello, Trans. Am. Math. Soc. 260, 1 (1980).

[9] F. L. Williams, J. Math. Phys. 38, 796 (1997).

[10] E. Elizalde, S. D. Odintsov, A. Romeo, A. A. Bytsenko and S. Zerbini, Zeta Regularization Techniques with Applications (World Scientific, Singapore, 1994).

[11] A. A. Bytsenko, G. Cognola, L. Vanzo and S. Zerbini,
Phys. Rept. 266, 1 (1996).

[12] A. A. Bytsenko, G. Cognola, E. Elizalde, V. Moretti and S. Zerbini, Analytic Aspects of Quantum Fields (World Scientific, Singapore, 2003).

[13] A. A. Bytsenko, L. Vanzo and S. Zerbini, Nucl. Phys. B 505, 641 (1997).

[14] A. A. Bytsenko, Nucl. Phys. (Proc. Suppl.) B 104, 127 (2002).

[15] A. A. Bytsenko, A. E. Gonçalves and F. L. Williams, Int. J. Mod. Phys. A 18, 2041 (2003).

[16] I. S. Gradshteyn and I. M. Ryzik, Table of Integrals, Series, and Products, Collected and Enlarged Edition Prepared by A. Jeffrey (Academic Press, 1980).

[17] A. A. Bytsenko, E. Elizalde and M. E. X. Guimarães, Int. J. Mod. Phys. A 18, 2179 (2003). 